\title{
Extension of Usable Spectral Range of Peltier Cooled Photodetectors
}

\author{
A. Piotrowski*, J. Piotrowski, W. Gawron, J. Pawluczyk and M. Pedzinska \\ Vigo System S.A., Poznańska 129/133, 05-850 Ożarów Maz., Poland
}

\begin{abstract}
This paper provides update on development of the Peltier cooled detectors optimized for wavelengths above $13 \mu \mathrm{m}$. Initially, the devices made by Vigo were mostly used for uncooled detection of $\mathrm{CO}_{2}$ laser radiation. Over the years the performance and speed of response has been steadily improved. At present the uncooled or Peltier cooled photodetectors can be used for sensitive and fast response detection in the mid-wavelength and long-wavelength infrared spectral range. The devices have found important applications in IR spectrometry, quantum cascade laser based gas analyzers, laser radiation alerters and many other IR systems. Recent efforts were concentrated on the extension of useful spectral range to $>13 \mu \mathrm{m}$, as required for its application in Fourier transform IR spectrometers. This was achieved with improved design of the active elements, use of monolithic optical immersion technology, enhanced absorption of radiation, dedicated electronics, series connection of small cells in series, and last but not least, applying more efficient Peltier coolers. Practical devices are based on the complex $\mathrm{HgCdTe}$ heterostructures grown on GaAs substrates with metal-organic chemical vapor deposition technique with immersion lens formed by micromachining in the GaAs substrates. The results are very encouraging. The devices cooled with miniature 4 stage Peltier coolers mounted in TO-8 style housings show significant response at wavelength exceeding $16 \mu \mathrm{m}$.
\end{abstract}

PACS numbers: 85.60.Gz, 82.80.Gk, 73.61.Ga, 78.66.Hf, 81.05.Dz

\section{Introduction}

Mid-wavelength infrared (MWIR) and long-wavelength infrared (LWIR) photodetectors operated without cryocooling are in demand for many important applications. For a long time the devices suffered from high dark currents and low junction resistances, and in consequence, in poor signal-to-noise performance. Recent considerations of the fundamental detector mechanisms suggest that, in principle, near perfect detection can be achieved in the MWIR and LWIR range without the need for cryogenic cooling [1-9]. To achieve this, thermal generation must be suppressed to possible lowest level. A number of concepts to improve performance of photodetectors operating at near room temperatures have been proposed including optimization of absorber material, reduction of the physical detector volume and suppression of thermal generation by non-equilibrium depletion of the absorber in charge carriers. Near-background limited infrared photodetector (near-BLIP) performance is now easily achievable in the Peltier cooled MWIR detectors. Peltier cooled devices LWIR devices operating up to $\approx 13 \mu \mathrm{m}$ wavelength were reported.

The main objectives of this IR detector research is to extend the useful spectral range of the Peltier cooled photodetectors to longer wavelengths while preserving

\footnotetext{
* corresponding author; e-mail: apiotrow@vigo.com.pl
}

near-BLIP performance and high speed of response. To achieve this we have followed the three-dimensional heterojunction device concept that has been developed at Vigo [1-3]:

- integration of the optical and detection function on one heterostructural chip;

- device structure optimized for the best compromise between requirements of a high quantum efficiency and a low dark current;

- monolithic optical immersion of detector structure to microlenses formed from the substrates of epitaxial layer;

- retroreflector to enhance absorption of IR radiation;

- formation of a low finesse optical resonance cavity due to reflections at the substrate-heterostructure interfaces, internal interfaces, and the heterostructure top surface.

\section{Growth}

The detector architecture used for backside illuminated heterojunction $\mathrm{HgCdTe}$ photodiode (Fig. 1) is a modified heterojunction $\mathrm{N}^{+} p \mathrm{P}^{+}$photodiode, developed by UK scientists [2], in which lightly doped narrow-gap absorber is sandwiched between wider gap heavily $n$ - and $p$-type 
doped layers that play a role of minority and majority carrier contacts. The structure is designed for mesa configuration, with relatively thick $\mathrm{N}^{+}$layer serving as the mesa base and playing also role of short wavelength cut-off window.

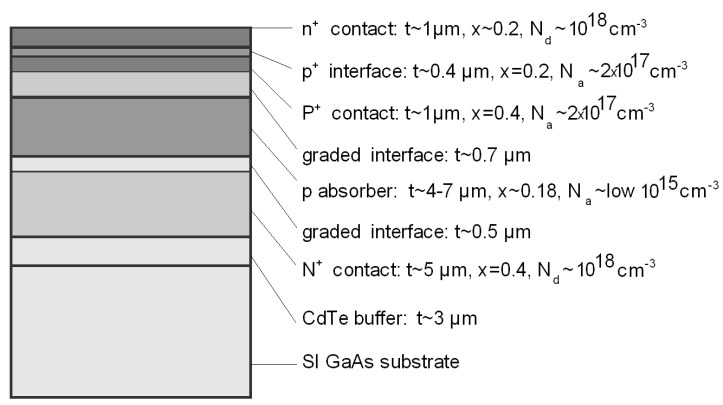

Fig. 1. Schematic layer structure of the heterojunction $\mathrm{HgCdTe}$ photodiode.

The main modifications in comparison to the standard three-layer $\mathrm{N}^{+} p \mathrm{P}^{+}$structure is programmed grading of band gap and doping level at interfaces between:

- Heavily doped $\mathrm{N}^{+}$layer and absorber in purpose to minimize thermal generation and tunneling currents, improve responsivity and reduce capacitance.

- Absorber and heavily doped $\mathrm{P}^{+}$layer in purpose to minimize minority carrier injection.

- Heavily doped $\mathrm{P}^{+}$layer and contact metalization to provide a low resistance contact and protect sensitive regions of the device during mechanical handling, metalization deposition and flip-chip mounting. The interface can be described as $p^{+} n^{+}$structure with composition close to that of the absorber.

The (111)B HgCdTe heterostructures have been grown on CdTe buffered (100) GaAs substrates using metal-organic chemical vapor deposition (MOCVD) technique $[6,7,10-15]$. MOCVD growth has been carried out in the horizontal reactor of the Aixtron AIX-200 reactor. Two separated gas inlet channels are provided to prevent premature gas reactions and dust formation. Hydrogen is used as a carrier gas. Dimethylcadmium (DMCd) and diisopropyltelluride (DIPTe) are used as precursors. Ethyl iodine (EI) and trisdimethylaminoarsenic (TDMAAs) were the donor and acceptor dopant sources. DMCd and EI are delivered through the one channel while DIPTe and TDMAAs through the other channel over the quartz container where elementary mercury is being held. The Aixtron gas foil rotation technique has been applied for better composition uniformity. High temperature annealing was used before each growth run for reactor cleaning. The typical growth conditions are $350^{\circ} \mathrm{C}$ deposition temperature, $200-220^{\circ} \mathrm{C}$ mercury source temperature and 500 mbar reactor pressure.

Stoichiometry control during growth by $\mathrm{Hg} / \mathrm{Cd}$-rich vapor treatment after each $\mathrm{HgTe}$ phase of interdiffused multilayer process (IMP) cycle $[7,15]$ has been applied to minimize vacancy concentration without any prolonged post-growth thermal annealing.

Composition and doping control of thick layers $\left(\mathrm{N}^{+}\right.$ and absorber) is relatively simple, based on selection of suitable parameters of IMP growth. The compositions of the two layers were determined comparing experimental and calculated data for the short and long wavelength cutoff spectra, respectively.

Grading of interfaces was controlled by continuous changes of parameters of consecutive IMP phases - their durations, precursors concentration and other factors to achieve required composition and doping profiles. The composition and doping profiles of the interfaces depend not only on growth parameters but also on composition and doping of adjacent layers. Further complications come from mutual and complex dependences of:

- $\mathrm{CdTe} / \mathrm{HgTe}$ interdiffusion on composition and arsenic doping level;

- arsenic diffusion on composition;

- As doping level on composition.

Secondary ion mass sepctroscopy (SIMS) proved to be an invaluable tool to determine composition and doping grading at interfaces. The known compositions of the $\mathrm{N}^{+}$ and absorber layers determined from photoresponse spectra are used as built-in standards for SIMS calibration. In practice, the IMP growth parameters are selected basing on measured profiles and performance of previously grown heterostructures. Recent experiments have shown strong dependence of dark current and responsivity on grading parameters at $\mathrm{N}^{+}$-absorber and absorber $/ \mathrm{P}^{+}$interfaces revealing great potential for further improvement of performance with growth refinement. Two problems were revealed:

- Increasing absorber composition towards contacts due to in-diffusion of CdTe. It was corrected by introducing more thick $\mathrm{HgTe}$ layer in the first and last IMP pairs of absorber.

- Excess doping of absorber and its interface caused by arsenic diffusion from the $\mathrm{P}^{+}$layer. The problem was solved with programmed doping of the $\mathrm{P}^{+}$ layer.

\section{Processing}

Mesa structures were fabricated by photolithography-assisted dry and wet etching. After etching the mesa structures were passivated by CdZnTe sputtering. Contact metallization was then deposited by sputtering of $\mathrm{Cr} / \mathrm{Au}$ layers using lift-off technique. Both lateral collection design and indium bump bonding has been used to contact mesa top layer; the first technique seems to be more reliable, however. 
Next, the wafers were diced into individual chips and immersion lens [16] were prepared from the GaAs substrates using numerically controlled turning machines (Fig. 2). The detector chips with immersion lenses were then flip-chip bonded to an $\mathrm{Au} / \mathrm{Cr}$ pattern on sapphire (Fig. 2) or ceramic cold fingers of the Peltier coolers (Fig. 3). Two-, three-, and four-stage Peltier coolers have been used.

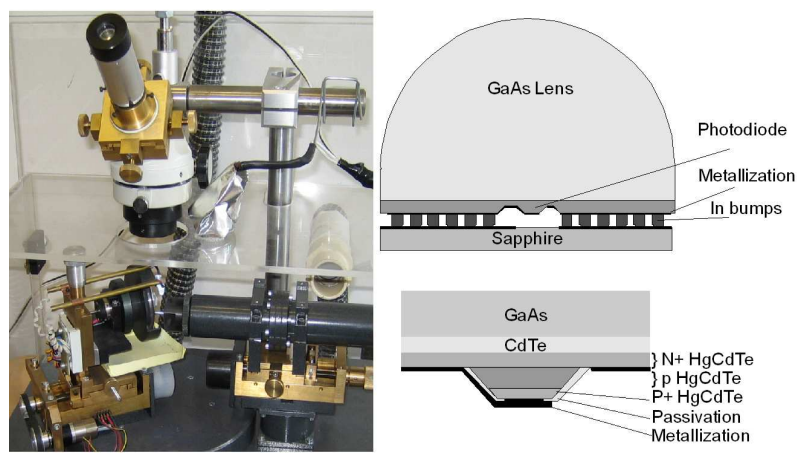

Fig. 2. Numerically controlled turning machine, schematic cross-section of optically immersed photodiode bonded to $\mathrm{Au} / \mathrm{Cr}$ pattern on sapphire and enlarged view of active element.

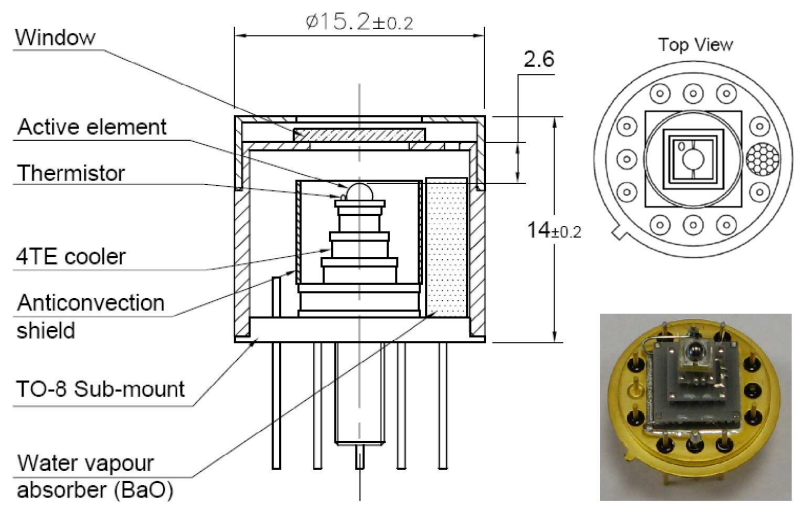

Fig. 3. Optically immersed detector mounted on cold finger on four-stage Peltier cooler.

The last stage of detector preparation is its hermetization in TO-8 based housing, backfilled with $\mathrm{Kr} / \mathrm{Xe}$ mixture. Uncoated and AR coated $\mathrm{ZnSe}$ was used as IR transmitting windows (Fig. 3). The two-, three-, and four-stage TE coolers fit to the same TO- 8 header; the only difference is the enclosure height for 4-stage cooler detectors by approximately $2 \mathrm{~mm}$ larger.

The housed devices have been typically integrated with associated electronics (readout transimpedance preamplifiers that provide also optimized bias to detectors, Peltier cooler controllers and $\mathrm{A} / \mathrm{D}$ converters) in detection modules. This makes them convenient to use and immune to electromagnetic interference (EMI), DC or transient overbias.
The devices have already found important applications in various LWIR systems. The examples are the miniaturized FTIR spectrophotometer being developed under the MEMFIS project, some quantum cascade laser based IR systems and other applications.

\section{Results}

Figure 4 shows measured spectral responsivity of $200 \mathrm{~K}$ cooled photoconductor and photovoltaic devices. Cooling to $200 \mathrm{~K}$ shifts cutoff wavelength above $12 \mu \mathrm{m}$. Significant response was observed in some devices at wavelength exceeding $16 \mu \mathrm{m}$. The responsivity significantly increases with reverse bias both in uncooled and cooled devices, saturating at reverse voltages exceeding approximately. $300 \mathrm{mV}$ and $100 \mathrm{mV}$ for uncooled and $200 \mathrm{~K}$ devices, respectively.

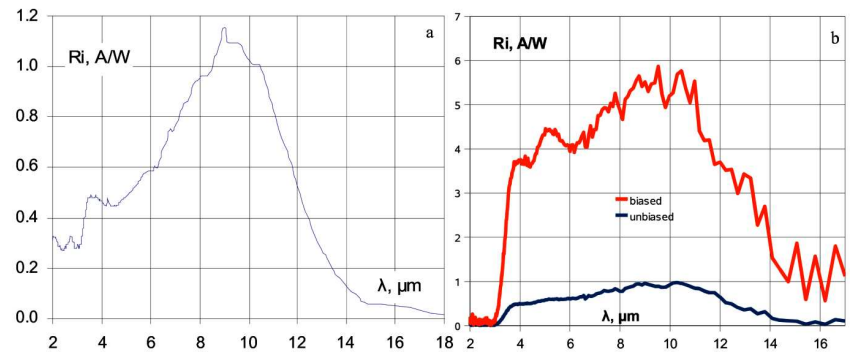

Fig. 4. Measured spectral responsivity of $200 \mathrm{~K}$ Peltier cooled (a) photoconductor, (b) photovoltaic devices.

The $I-V$ plot of the photovoltaic detector measured at $200 \mathrm{~K}$ (Fig. 5) shows three different regions:

- linear $I-V$ at low $(>+0.1 \mathrm{~V}$ to $-0.08 \mathrm{~V})$ bias,

- current saturation for voltages $>-0.17 \mathrm{~V}$ and less than $0.08 \mathrm{~V}$,

- tunnel-like current for large reverse bias.

As size analysis has shown, the reverse bias dark current at $200 \mathrm{~K}$ is proportional to the device area indicating insignificant role of surface. This was true both for the saturation and tunnel-like dark current range. Closer examination of dark current dependences indicates that the linear range at low voltages is due to series resistance while the dark current in the saturation range is the diffusion dark current generated in absorber by the Auger or Shockley-Read-Hall mechanism.

The shot noise detectivities measured for many optically immersed devices at frequencies above the $1 / f$ noise knee $(\approx 1 \mathrm{MHz})$ are quite large; exceeding $10^{9} \mathrm{~cm} \mathrm{~Hz}^{1 / 2} \mathrm{~W}$ at $15 \mu \mathrm{m}$. Further red shift of spectral response and significant increase of performance at $16 \mu \mathrm{m}$ was observed with cooling to $180 \mathrm{~K}$, achievable with the same 4-stage coolers mounted in vacuum detector packages. 


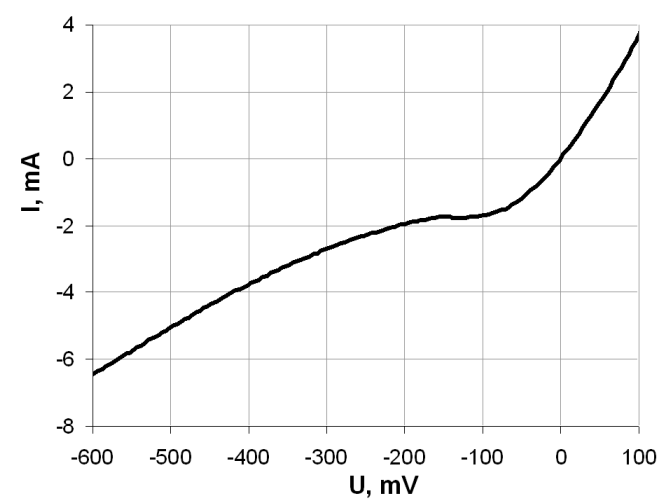

Fig. 5. Measured $I-V$ plot at $200 \mathrm{~K}$ (optical area of $\left.0.5 \times 0.5 \mathrm{~mm}^{2}\right)$.

Large changes of dark current and responsivity have been observed varying grading parameters of both absorber revealing great potential for further improvement of performance with growth refinement. The results will be published soon.

Black body based optical test bench was used to observe saturation effects. Figure 6 shows signal versus optical power on the detector compared to linear detector. Photoconductors (PC) tend to saturate at $\approx 2 \mathrm{~mW} / \mathrm{mm}^{2}$ optical power densities while for photovoltaic $(\mathrm{PV})$ detectors saturation threshold typically exceeds $2.5 \mathrm{~mW} / \mathrm{mm}^{2}$. Digital or analog linearization system was used to increase the dynamic range to $\approx 5 \mathrm{~mW} / \mathrm{mm}^{2}$ in some devices.

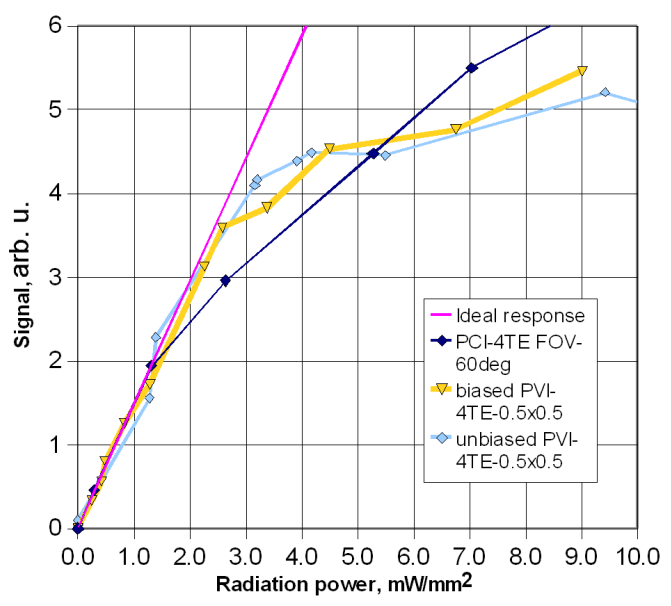

Fig. 6. Saturation effects for PC, unbiased PV and biased PV detectors.

\section{Summary}

Useful spectral range of $\mathrm{HgCdTe}$ Peltier cooled photodiodes has been extended to $\approx 16 \mu \mathrm{m}$ with opti- mized $\mathrm{HgCdTe}$ heterostructure, monolithic optical immersion of active elements to hyperhemispherical microlenses formed from GaAs substrates, more efficient Peltier coolers and other solutions. Further work is needed to optimize composition and doping profiles at absorber interfaces with great promise for significant performance improvement.

\section{Acknowledgments}

This work was partially supported by the Ministry of Science and Higher Education under project No. PBZ-MNiSW 02/I/2007.

\section{References}

[1] J. Piotrowski, W. Galus, M. Grudzien, Infrared Phys. 31, 1 (1990).

[2] T. Elliott, Semicond. Sci. Technol. 5, S30 (1990).

[3] T. Elliott, N.T. Gordon, A.M. White, Appl. Phys. Lett. 74, 2881 (1999).

[4] M.A. Kinch, J. Electron. Mater. 29, 809 (2000).

[5] N.T. Gordon, R.S. Hall, C.L. Jones, C.D. Maxey, N.E. Metcalfe, R.A. Catchpole, M. White, J. Electron. Mater. 29, 818 (2000).

[6] A. Piotrowski, P. Madejczyk, W. Gawron, K. Kłos, M. Romanis, M. Grudzień, A. Rogalski, J. Piotrowski, Opto-Electron. Rev. 12, 453 (2004).

[7] A. Piotrowski, W. Gawron, K. Klos, J. Pawluczyk, J. Piotrowski, P. Madejczyk, A. Rogalski, Proc. SPIE 5957, 59570J (2005).

[8] J. Piotrowski, A. Rogalski, High-Operating-Temperature Infrared Photodetectors, SPIE, Bellingham 2007.

[9] M.A. Kinch, Fundamental of Infrared Detector Materials, SPIE Press, Bellingham 2007.

[10] A. Piotrowski, P. Madejczyk, W. Gawron, K. Klos, M. Romanis, M. Grudzien, J. Piotrowski, A. Rogalski, Bul. Pol. Acad. Tech. 53, 139 (2005).

[11] A. Piotrowski, P. Madejczyk, W. Gawron, K. Kłos, J. Pawluczyk, M. Grudzień, J. Piotrowski, A. Rogalski, Proc. SPIE 5732, 273 (2005).

[12] P. Madejczyk, A. Piotrowski, W. Gawron, K. Kłos, J. Pawluczyk, J. Rutkowski, J. Piotrowski, A. Rogalski, Opto-Electron. Rev. 13, 239 (2005).

[13] A. Piotrowski, J. Piotrowski, Proc. SPIE 6206, 62060T (2006).

[14] A. Piotrowski, P. Madejczyk, W. Gawron, K. Kłos J. Pawluczyk, J. Rutkowski, J. Piotrowski, A. Rogalski, Infrared Phys. Technol. 49, 173 (2007).

[15] A. Piotrowski, K. Kłos, J. Electron. Mater. 36, 1052 (2007).

[16] M. Grudzien, J. Piotrowski, Infrared Phys. 29, 251 (1989). 